\title{
MRI texture analysis (MRTA) of T2-weighted images in Crohn's disease may provide information on histological and MRI disease activity in patients undergoing ileal resection
}

\author{
Jesica Makanyanga ${ }^{1} \cdot$ Balaji Ganeshan $^{2} \cdot$ Manuel Rodriguez-Justo $^{3} \cdot$ \\ Gauraang Bhatnagar ${ }^{1} \cdot$ Ashley Groves $^{2} \cdot$ Steve Halligan $^{1} \cdot$ Ken Miles $^{2}$ • \\ Stuart A. Taylor ${ }^{1}$
}

Received: 6 May 2015 /Revised: 29 February 2016 / Accepted: 8 March 2016 /Published online: 5 April 2016

(C) The Author(s) 2016. This article is published with open access at Springerlink.com

\begin{abstract}
Objectives To associate MRI textural analysis (MRTA) with MRI and histological Crohn's disease (CD) activity.

Methods Sixteen patients (mean age 39.5 years, 9 male) undergoing MR enterography before ileal resection were retrospectively analysed. Thirty-six small $(\leq 3 \mathrm{~mm})$ ROIs were placed on T2-weighted images and locationmatched histological acute inflammatory scores (AIS) measured. MRI activity (mural thickness, T2 signal, T1 enhancement) (CDA) was scored in large ROIs. MRTA features (mean, standard deviation, mean of positive pixels (MPP), entropy, kurtosis, skewness) were extracted using a filtration histogram technique. Spatial scale filtration (SSF) ranged from 2 to $5 \mathrm{~mm}$. Regression (linear/logistic) tested associations between MRTA and AIS (small ROIs), and $\mathrm{CDA} /$ constituent parameters (large ROIs).
\end{abstract}

Electronic supplementary material The online version of this article (doi:10.1007/s00330-016-4324-4) contains supplementary material, which is available to authorized users.

Stuart A. Taylor

csytaylor@yahoo.co.uk

1 Center for Medical Imaging, University College London and University College London Hospitals NIHR Biomedical Research Centre, 250 Euston Road, London NW1 2BU, UK

2 Institute of Nuclear Medicine, University College London, University College Hospital, 235 Euston Road, London NW1 2BU, UK

3 Department of Research Pathology, University College London Cancer Institute, University College London, Rockefeller Building, London WC1E 6JJ, UK
Results Skewness ( $\mathrm{SSF}=2 \mathrm{~mm}$ ) was associated with AIS [regression coefficient (rc) 4.27, $p=0.02$ ]. Of 120 large ROI analyses (for each MRI, MRTA feature and SSF), 15 were significant. Entropy $(\mathrm{SSF}=2,3 \mathrm{~mm})$ and kurtosis $(\mathrm{SSF}=3 \mathrm{~mm}$ ) were associated with $\mathrm{CDA}$ ( $\mathrm{rc} 0.9,1.0,-0.45$, $p=0.006-0.01)$. Entropy and mean ( $\mathrm{SSF}=2-4 \mathrm{~mm})$ were associated with T2 signal [odds ratio (OR) 2.32-3.16, $p=0.02-$ 0.004 ], [OR 1.22-1.28, $p=0.03-0.04]$. MPP (SSF $=2 \mathrm{~mm})$ was associated with mural thickness (OR 0.91, $p=0.04$ ). Kurtosis $(\mathrm{SSF}=3 \mathrm{~mm}$ ), standard deviation $(\mathrm{SSF}=5 \mathrm{~mm}$ ) were associated with decreased T1 enhancement (OR 0.59, 0.42, $p=0.004,0.007)$.

Conclusions MRTA features may be associated with CD activity.

Key Points

- MR texture analysis features may be associated with Crohn's disease histological activity.

- Texture analysis features may correlate with MR-dependent Crohn's disease activity scores.

- The utility of MR texture analysis in Crohn's disease merits further investigation.

Keywords Crohn disease $\cdot$ Magnetic resonance imaging . Textural analysis $\cdot$ Small intestine $\cdot$ Histology
Abbreviations
CDAS Crohn's disease activity score
LoG Laplacian of Gaussian
MaRIA Magnetic resonance index of activity
MRTA MRI textural analysis
SSF Spatial scale of the filter 


\section{Introduction}

MR enterography is established for assessment of disease activity in small bowel and colonic Crohn's disease. Mural features such as thickness [1-3], T2 signal intensity [3, 4], and contrast enhancement [1-4] are significantly correlated with endoscopic and histological disease activity and MRI is used routinely to assesses global disease burden [5] and monitor treatment response [6].

Texture analysis (TA) is a novel image analysis technique that can quantify image heterogeneity resulting from changes not appreciated by the human eye [7, 8]. Disease processes may introduce image heterogeneity and TA has been studied in many cancers including lung [9], breast [10], and colorectal [11]. The underlying rationale is that complex microscopic tumour heterogeneity resulting in structures of different sizes and variation may be reflected indirectly by the distribution of greyscale levels and/or pixel intensity on diagnostic images such as CT and MRI (i.e. macroscopic heterogeneity). In cancer, changes in image texture are associated with hypoxia, angiogenesis, cellular proliferation, tumour grade, genetic mutation status $[12,13]$, and with prognosis and treatment response $[14,15]$.

The hallmark of active Crohn's disease on histology includes transmural inflammation, fissuring ulcers, submucosal expansion/oedema, and inflammation-driven new vessel formation [16]. It is plausible that these may be reflected by texture changes so that texture analysis could refine the use of MRI as a biomarker of disease activity further.

One sequence ubiquitous to MR enterography protocols is T2-weighted imaging. Radio-pathological comparisons using surgical specimens have shown significant linear correlations between mural T2 signal and inflammatory activity [4, 17, 18], and assessment of T2 signal is common to MRI activity scores such as the MaRIA [1] and Crohn's disease activity scores [19].

The aim of our study was to explore whether MRTA measured in T2-weighted MRI images is associated with histological and MRI scores of disease activity.

\section{Materials and methods}

\section{Study population}

The institutional review board issued a waiver for reconsenting patients for additional data analysis. A retrospective analysis was undertaken using data collated between July 2006 and December 2007 from 18 (mean age 31 years, 9 male) consecutive patients with proven Crohn's disease (based on standard clinical, endoscopic, and histological criteria) undergoing MR enterography and scheduled to undergo elective small bowel resection for disease-related complications [17].

The original study aimed to validate proposed MR enterography features of Crohn's disease activity using a transmural histopathological reference standard. The current study used the collated MRI data sets and same histopathological reference standard and additionally applied MRTA to axial T2-weighted images. Two patients from the original study were excluded because axial T2-weighted images were unavailable for analysis because movement artefact degraded the image quality.

The Montreal classification was recorded for each patient. Harvey Bradshaw index and C-reactive protein were also recorded within the 5 days preceding surgery (Table 1).

\section{MR enterography protocol}

Full details of the MR enterography protocol and subsequent matching of sites of histological sampling to preoperative MR enterography were as described previously [17]. Patients underwent MR enterography within 2 weeks of surgery (mean 4 days, range $1-13$ days) on a 1.5-T MRI unit (Avanto; Siemens, Erlangen, Germany)

As part of the standard MR enterography protocol, axial single shot turbo spin echo (SSTSE) sequences were obtained (TR $800 \mathrm{~ms}$, TE $86 \mathrm{~ms}$, matrix $256 \times 195$ ) together with preand post-contrast T1 VIBE images (Table 2)

Within $24 \mathrm{~h}$ of surgery, the resected specimen was pinned to a board in its anatomical orientation and scanned using an SSTSE sequence in axial and coronal planes.

Table 1 Demographic characteristics of included patients

\begin{tabular}{llcc}
\hline Age & Montreal classification & Harvey Bradshaw index & CRP $(\mathrm{mg} / \mathrm{L})$ \\
\hline 17 & A1L3B1 & 7 & 27 \\
16 & A1L3B3 & 5 & 157 \\
21 & A2L3B2 & 8 & 7 \\
26 & A2L3B2 & 4 & $<5$ \\
28 & A2L3B1 & 4 & 84 \\
16 & A1L3B3p & 8 & 30 \\
40 & A1L3B2 & 17 & 107 \\
51 & A2L3B2 & 6 & 113 \\
40 & A2L3B2 & 4 & 70 \\
27 & A1L3B1 & 16 & 66 \\
49 & A3L3B1 & 7 & 142 \\
19 & A1L3B2 & 4 & 19 \\
56 & A3L3B1 & 4 & 184 \\
47 & A3L1B1 & 15 & 70 \\
16 & A2L3B1 & 6 & 114 \\
29 & A2L3B1 & 10 & \\
\hline
\end{tabular}


Table 2 MR imaging parameters for preoperative patient imaging and post-resection specimen imaging

\begin{tabular}{|c|c|c|c|c|c|}
\hline \multirow[b]{2}{*}{ Parameter } & \multicolumn{4}{|l|}{ Clinical scan } & \multirow{2}{*}{$\begin{array}{l}\text { Specimen scan } \\
\text { Half-Fourier } \\
\text { RARE sequence }\end{array}$} \\
\hline & $\begin{array}{l}\text { Coronal and axial half-Fourier } \\
\text { RARE sequence }\end{array}$ & $\begin{array}{l}\text { Coronal and axial } \\
\text { True FISP sequence }\end{array}$ & $\begin{array}{l}\text { Baseline VIBE } \\
\text { sequence }\end{array}$ & $\begin{array}{l}30 \text {-s and } 70 \text {-s post } \\
\text { contrast VIBE sequence }\end{array}$ & \\
\hline Field of view (mm) & Variable & Variable & Variable & Variable & Variable \\
\hline No. of sections & $20 / 26$ & $25 / 34$ & 48 & 48 & 15 \\
\hline No. of stacks & $1 / 4$ & $1 / 2$ & 1 & 1 & 1 \\
\hline Repetition time (ms) & $1200 / 800$ & $4 / 4.2$ & 7.2 & 7.2 & 1200 \\
\hline Echo time (ms) & $86 / 86$ & $1.7 / 2.1$ & 2.4 & 2.4 & 84 \\
\hline Image matrix & $256 \times 195$ & $256 \times 205$ & $256 \times 135$ & $256 \times 135$ & $256 \times 195$ \\
\hline Section thickness (mm) & $4 / 4$ & $4 / 4$ & 3 & 3 & 2 \\
\hline Section gap (mm) & $5.2 / 4.2$ & $5.2 / 5.4$ & 0 & 0 & 0 \\
\hline No. of measures acquired & 1 & 1 & 1 & 1 & 1 \\
\hline Turbo factor & 195 & 1 & 1 & 1 & 195 \\
\hline $\begin{array}{l}\text { Integrated parallel } \\
\text { acquisition technique }\end{array}$ & GRAPPA factor of 2 & NA & NA & NA & $\begin{array}{l}\text { GRAPPA } \\
\text { factor of } 2\end{array}$ \\
\hline Flip angle $\left(^{\circ}\right)$ & 50 & 46 & 10 & 10 & 150 \\
\hline
\end{tabular}

The study coordinator, in collaboration with the operating surgeon, reviewed the preoperative MR enterography to locate the exact segment of bowel resected (using fixed anatomical landmarks such as the ileo-caecal valve (ICV), site of fistula etc.) and then chose one to five sampling sites (median, three) through the resected bowel on the preoperative MRI scan for detailed histological correlation.

The coordinator in consensus with the study histopathologist (the latter with 15 years of experience in gastrointestinal histopathology) then located the selected sampling sites on the resected specimens. Sampling sites were co-located between MRI scan and the specimen using distance measurements with reference to fixed anatomical structures such as the ICV, fistula site, abscess etc.

\section{MRI Crohn's disease activity (CDA) scoring}

The preoperative MR enterography images were uploaded onto a standard picture archiving and communication system workstation (Agfa Healthcare UK, Brentwood, England). Segmental disease activity was scored in consensus by two gastrointestinal radiologists with 10 and 15 years' experience respectively of MR enterography using the MR Crohn's disease activity (CDA) score [19], previously validated against a histological reference standard. Specifically, activity in the region of all sampling sites in the subsequently resected segment was scored on a scale 0 to 3 for mural thickness, mural T2 signal, perimural T2 signal, and qualitative mural enhancement (maximum score =12) [19] (see electronic supplementary material). The individual scores were summed to give the CDA score for each site. If a particular sampling site had a range of activity based on CDA scoring in the vicinity, the area attracting the highest CDA score was used for subsequent textural analysis.

\section{Region of interest placement and MR textural analysis}

Axial T2-weighted images were uploaded into proprietary software for textural analysis (TexRAD, www.texrad.com, part of Feedback Plc, Cambridge, UK) [7].

Two regions of interest (ROI) types ("large" and "small") were drawn freehand by two observers in consensus (a radiologist with 15 years of experience of MRI enterography and a research fellow with 2 years' experience of MRI enterography). Observers were blinded to any histological analysis (other than the exact site of sectioning) but had full access to the complete MRI data sets including coronal T2and T1-weighted images.

The observers drew a small ROI in each of the sampling sites previously identified for detailed histopathogical matching as described above. All available MRI sequences and orientations were used to locate these designated sites on the axial T2-weighted image. A small ROI was placed at each site. Observers were instructed to include the full bowel wall thickness within the ROI but to limit its width to $3 \mathrm{~mm}$ so as to match the exact site of histological sampling as closely as possible (Fig. 1a).

The observers then scrolled through the axial T2-weighted sequence and selected the slice which had been used to grade the MRI CDA as described above. A large ROI was drawn to include as much of the diseased segment as possible in this single slice. Care was taken to exclude any intraluminal or mesenteric tissue (Fig. 1b). 

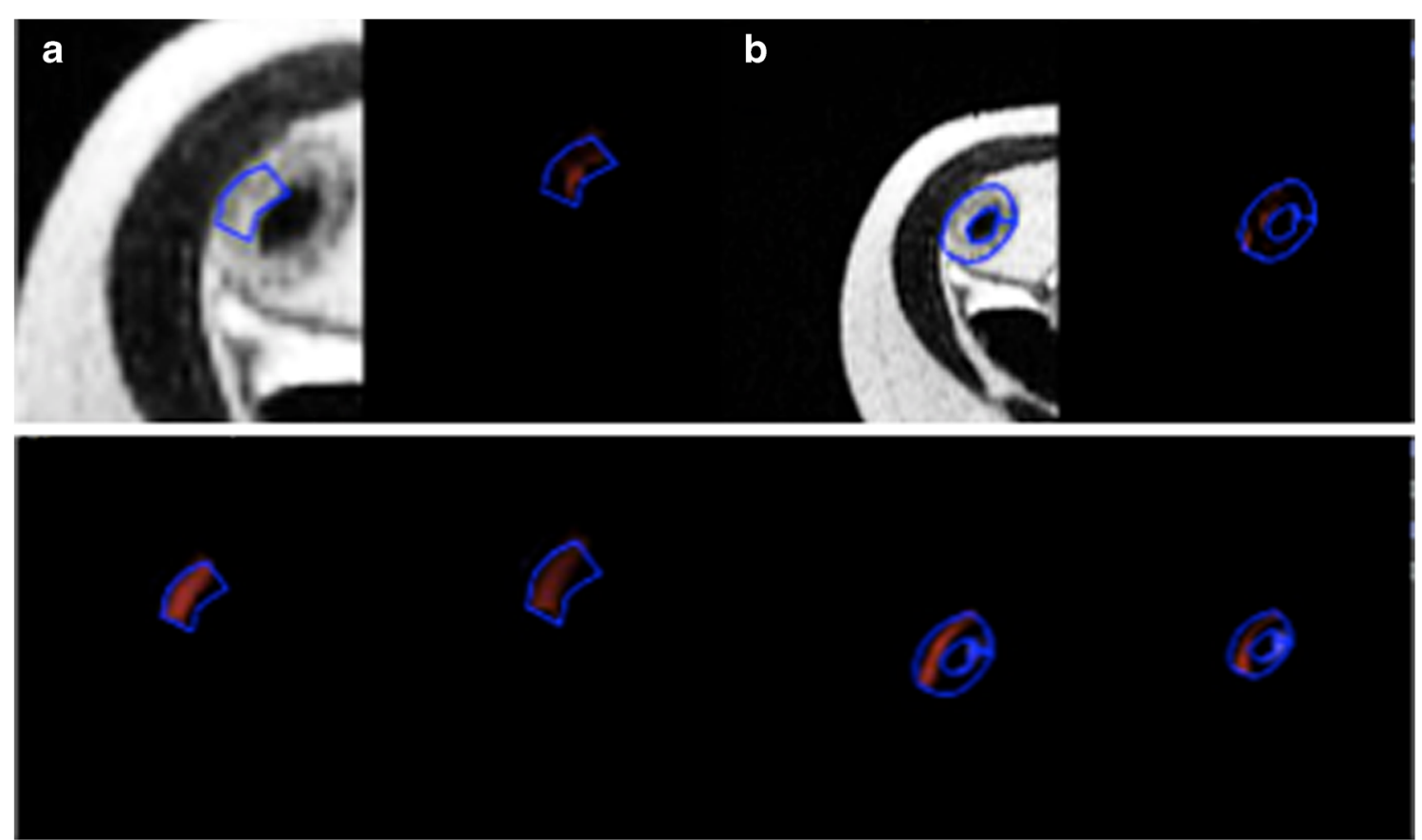

Fig. 1 Examples of small (a) and large (b) regions of interest drawn on axial half-Fourier RARE sequence images in diseased bowel subsequently resected along with the texture maps at fine (upper right panel),

There were a total of 36 small ROIs and 36 large ROIs across the 16-patient cohort.

Finally, a further ROI was placed in the cerebrospinal fluid (CSF) in the same slice for normalization of textural parameters on the $\mathrm{T} 2$ weighted image (see below).

\section{Texture analysis}

Textural analysis was performed for both ROI types using methodology previously described [10] and employing a filtration-histogram technique. Filtration extracts and enhances texture features at different sizes within ROIs and is followed by histogram quantification. MRTA was carried out using commercially available research software (TexRAD www.texrad.com, Feedback Plc, Cambridge, UK) [7]. Specifically the initial filtration step employs a Laplacian of Gaussian (LoG) bandpass filtration, which extracts and highlights image features of different sizes corresponding to the spatial scale of the filter (SSF), ranging between $2 \mathrm{~mm}$ (fine textural features) and $6 \mathrm{~mm}$ (coarse features) in width (radius). Quantification of histograms (with and without filtration) was based on mean (average value of the pixels within the ROI), standard deviation (width of the histogram or degree of variation/dispersion from the average), skewness (symmetry of the distribution), mean of positive pixels (MPP, average of the pixel values which are positive), kurtosis (pointiness of the distribution), and entropy (with higher values indicating increasing image irregularity or complexity). A summary of the premise underlying the interpretation of TA parameters is as medium (lower left panel), and coarse (lower right panel) texture scales. Small ROI $=42$ pixels. Large ROI $=169$ pixels

follows. The mean changes approximately in proportion to the number of objects highlighted and their mean brightness (dark objects are negative). The SD increases approximately in proportion to the square root of the number of objects highlighted by the filter and their mean intensity difference compared to background tissue (i.e. dark and bright objects are both positive); generally a higher SD value implies increased heterogeneity.

Skewness reflects the average brightness of highlighted objects (predominantly bright objects give positive skewness values and predominantly dark objects give negative skewness values). Skewness tends to zero with increasing number of objects highlighted and moves away from zero with intensity variations in highlighted objects.

Kurtosis is inversely related to the number of objects highlighted (whether bright or dark) and is increased by intensity variations in highlighted objects.

Because the gain factor for T2-weighted image acquisition can change between patients, the mean, SD, and MPP texture parameters (parameters potentially affected by this variation in gain factor) were normalized by dividing by the signal intensity of the CSF for each patient and at each filter SSF value.

\section{Histopathological assessment}

Histological analysis was performed by a specialist gastrointestinal pathologist (with 15 years of experience, blinded to other information). Sections were stained with haematoxylin- 
eosin (H\&E). At the specific sampling sites previously matched between the preoperative MRI and resected specimen (and corresponding to the placement of small ROI), acute inflammation was assessed on the basis of the method of Borley et al. [20] and an single acute inflammatory score (AIS) was calculated (electronic supplementary material).

\section{Statistical analysis}

The primary analysis was to examine the association between each textural parameter and histological score of activity (AIS) for the matched small ROI.

Secondary analyses examined the association between textural parameters and the MRI activity score (and its mural components) using the large ROI.

Separate analyses were performed for each filter. Given the limited size of the small ROI, only $\mathrm{SSF}=0,2$, and $3 \mathrm{~mm}$ were examined. For the large ROI analysis all filters $(0,2,3,4$, and $5 \mathrm{~mm}$ ) were applied.

All analyses used regression methods. Ordered logistic regression was used for the ordinal outcomes. AIS and total MRI score were both normally distributed and linear regression was used for these continuous outcomes. To account for the range of data for each textural parameter, odds ratios were expressed as follows: 50-unit increase (mean, SD, MPP), 1unit increase (skewness, entropy, and kurtosis), and 10-unit increase (MRI CDA score). Fischer's exact test was used to compare paired proportions as appropriate.

Within each patient there was more than one ROI analysed, i.e. there were multiple measurements per patient that therefore were non-independent. Robust standard errors (Huber White) were used to account for this. The level of significance was defined as $p<0.05$ for all analyses.

\section{Results}

Mean patient age was 39.5 years (range 16-66 years). Full demographic characteristics are in Table 1.

The range of MRTA parameters according to ROI size is shown in Table 3.

\section{MRTA and histological activity scores (small ROI)}

The mean size of the small ROI was 52 pixels, SD 23.5 (range 19-118).

The mean AIS was 4.53, SD 3.6 (range 0-11).

There was a significant positive correlation between skewness at $\mathrm{SSF}=2 \mathrm{~mm}$ and histological AIS [regression coefficient 4.27 (95\% CI 0.74, 7.79), $p=0.02$ ] (Fig. 2).

At SSF $=2 \mathrm{~mm}, 10$ of 23 small ROIs had an AIS $\leq 4$ of which $4(40 \%)$ exhibited skewness $\geq 0$. Conversely 13 small
ROIs had an AIS $>4$ of which $11(85 \%)$ exhibited skewness of $\geq 0$ ( $p=0.04)$ (Fig. 3).

There were no other significant associations between textural parameters and AIS $(p=0.261-0.418)$.

\section{MRTA and MRI activity score (large ROI)}

The mean size of the large ROI was 238 pixels, SD 107.7 (range 90-454).

The mean MRI CDA score was 8.8, SD 3.6 (range 3-14). A summary of the significant associations between texture parameters and mural MRI features according to SSF is shown in Table 4. For the large ROI, 15 of 120 associations (4 MRI scores, 6 MRTA parameters, and 5 filter levels) analysed were significant.

There were significant positive correlations between mean intensity values at different filter values (SSF 2, 3, and $4 \mathrm{~mm}$ ) and $\mathrm{T} 2$ signal score [greatest significance at $\mathrm{SSF}=2 \mathrm{~mm}$, odds ratio (OR) 1.22 (1.02-1.47), $p=0.03$ ] (Fig. 4, Table 4).

There were also associations between mural enhancement grade and MPP, kurtosis, and SD at several filter levels (Table 4). MPP in particular was significantly associated at filter values SSF 2,3 , and $5 \mathrm{~mm}$, [OR $0.91(0.86-0.97)$, $p=0.003 ;$ OR $0.86(0.75-0.99), p=0.04 ;$ and OR 0.18 $(0.07-0.48), p=0.001$, respectively]. In general the enhancement grade tended to decrease for a rise in MPP.

There was a significant correlation between normalized MPP $(\mathrm{SSF}=2 \mathrm{~mm})$ and decreasing mural thickness [OR 0.91 (95\% CI 0.83, 1.00), $p=0.04]$.

Mean and entropy were positively associated with mural T2 signal. Entropy in particular was correlated with mural T2 signal intensity at SSF 2,3 , and $4 \mathrm{~mm}$ (OR 2.32-3.16, $p=0.02-0.004$ ).

A significant correlation was demonstrated between total MRI CDA score and entropy ( $\mathrm{SSF}=2 \mathrm{~mm}$ and $3 \mathrm{~mm}$ ), regression coefficient $1.00(0.34,1.65)$, and $0.90(0.22,1.58)$ $(p=0.006, p=0.01)$, respectively. Kurtosis (SSF $3 \mathrm{~mm}$ ) was negatively associated with MRI CDA, regression coefficient $-0.45(-0.70,-0.19)(p=0.002)$.

\section{Discussion}

Based mainly on applications in cancer imaging, it is known that MRTA can reflect underlying histological phenotypes. Data from the present study suggests that MRTA may also reflect inflammatory phenotype in Crohn's disease.

Following careful matching of ROI size and positioning with the site of histological sampling, we found a significant positive correlation between pixel skewness and the histological acute inflammatory score at filter level $2 \mathrm{~mm}$. In support, the number of small ROIs at SSF $=2 \mathrm{~mm}$ with skewness $\geq 0$ was significantly greater for those with an AIS exceeding 4 , 
Table 3 Mean and range of values for each MR textural analysis parameter for small and large ROIs

\begin{tabular}{|c|c|c|c|c|c|c|}
\hline SSF & Mean (range) & SD (range) & Entropy (range) & MPP (range) & Skewness (range) & Kurtosis (range) \\
\hline \multicolumn{7}{|c|}{ Small ROI } \\
\hline 0 & $389.6(195.4-580.3)$ & $119.4(52.4-343.9)$ & $3.7(2.9-4.4)$ & $389.6(195.4-580.3)$ & $1.0(-0.2$ to 2.8$)$ & $1.2(-1.3$ to 12.1$)$ \\
\hline 2 & $-142.7(-582.5$ to 269$)$ & $146.3(0-467.5)$ & $1.8(0-3.5)$ & $89.2(0-269)$ & $0.1(-0.4$ to 0.8$)$ & $-1.0(-2.0$ to -0.3$)$ \\
\hline 3 & $-189.9(-688.5$ to 134.2$)$ & $82.5(0-214)$ & $1.6(0-3.4)$ & $0(0-178.2)$ & $0(-0.8$ to 1.2$)$ & $-1.0(-2$ to 0.0$)$ \\
\hline \multicolumn{7}{|c|}{ Large ROI } \\
\hline 0 & $400.5(217.3-548.5)$ & $135.5(49.7-324.3)$ & $4.9(4.1-5.3)$ & $400.5(217.3-548.5)$ & $1.2(-0.8$ to 2.7$)$ & $2.2(-1.1$ to 9.4$)$ \\
\hline 2 & $-121.5(-612.5$ to 128.2$)$ & $185.9(0-793.7)$ & $3.9(0-5.2)$ & $125.4(0-1559.5)$ & $0.1(-1.0$ to 1.9$)$ & $-0.0(-1.0$ to 7.0$)$ \\
\hline 3 & $-225.3(-549.5$ to 45.6$)$ & $161.9(0-850.4)$ & $3.6(0-5.2)$ & $97.2(0-1190.5)$ & $0.2(-0.8$ to 3.1$)$ & $-0.3(-1.4$ to 10$)$ \\
\hline 4 & $-189.1(-509$ to 2.9$)$ & $91.6(0-415.1)$ & $2.4(0-4.9)$ & $35.1(0-438.2)$ & $0(-1.2$ to 1.1$)$ & $-0.5(-1.7$ to 0.4$)$ \\
\hline 5 & $-160.4(-471.5$ to 11.8$)$ & $74.6(0-279.9)$ & $1.8(0-4.9)$ & $0(0-293.5)$ & $0.2(-1.2$ to 1.2$)$ & $-0.7(-1.2$ to 0.9$)$ \\
\hline
\end{tabular}

compared to those with less active disease (AIS lower than 4). Filtered skewness provides pixel distributional information on the filtered image and skewness $\geq 0$ reflects a preponderance of bright objects in the original MRI T2 image. It is therefore interesting to speculate as to what this relationship reflects. In T2-weighted MR images, the signal intensity is derived mainly from intracellular and extravascular extracellular space. Crohn's disease activity both at endoscopy and histology is associated with higher T2 signal intensity, likely in part reflecting acute inflammatory infiltration and oedema [16], but given this we may have expected unfiltered texture parameters (skewness, mean intensity value, and MPP) to be significantly associated with the histological activity score. It seems likely the association between histological activity and simple unfiltered texture parameters on an T2 weighted image is therefore an overly simplistic way of assessing activity. Fine $(2 \mathrm{~mm})$ filtered texture features (skewness) are perhaps more sensitive and likely to reflect the subtle bright (e.g. water and fat) and dark (e.g. fibrosis) features linked to underlying histological phenotype. Skewness has been associated with angiogenesis in several neoplasms [7, 9, 21]. Furthermore, in the

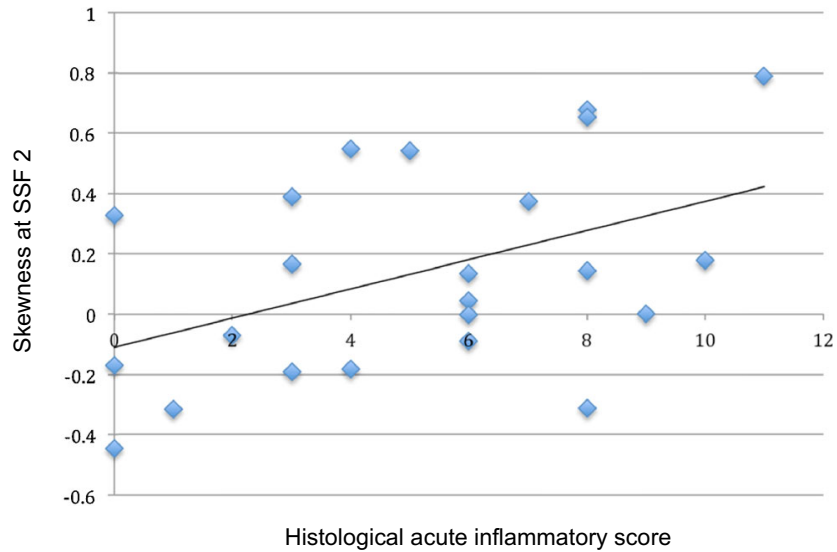

Fig. 2 Scatter plot and line of regression between histological acute inflammation score (AIS) in the bowel wall and skewness at SSF 2 for the small ROI large ROI there was an association between mural enhancement and MPP on T2 weighted images, supporting the concept that T2 signal is influenced by tissue vascularity in $\mathrm{CD}$. By using T2-weighted images rather than contrast-enhanced sequences we are of course limited with regard to the amount of information we can infer regarding inflammation-driven neoangiogenesis (which is well described in Crohn's disease [22]). Nevertheless, pixel distributions in a CD segment on T2-weighted images will be influenced by many parameters including inflammatory cellular infiltration, cellular and interstitial oedema, and blood vessel density and distribution.

Although most work in textural analysis has concentrated on neoplasia and used $\mathrm{CT}$, recent data also suggests textural changes on MRI reflect inflammatory damage in brain, skeletal muscle, and cartilage [23-25]. Indeed, Mahapatra et al. recently reported the use of textural features such as skewness and kurtosis on post-contrast-enhanced T1-weighted images to semi-automatically locate bowel afflicted by Crohn's disease [26]

Although use of the large ROI and comparison with an MRI CDA is inferior to detailed histopathogical correlation, such an approach can provide a noninvasive insight regarding interpretation and potential utility of TA in MR enterography. Furthermore, MRTA may have a role by providing a more objective measurement of signal values rather than subjective grading by radiologists, who are known to suffer interobserver variation [27].

Entropy is a measure of signal irregularity within the ROI and was positively associated with T2 signal at three filter levels. Entropy was also positively associated with the MRI CDA score (although this does include a score of T2 signal, so the two observations are not independent). "Chaotic" and complex histology in active $\mathrm{CD}$ (reflected by image heterogeneity or complexity) would contrast with the more uniform appearances of chronic fibrotic disease, which would intuitively provide a link between signal entropy and activity. It is, however, notable that we found no such association using 


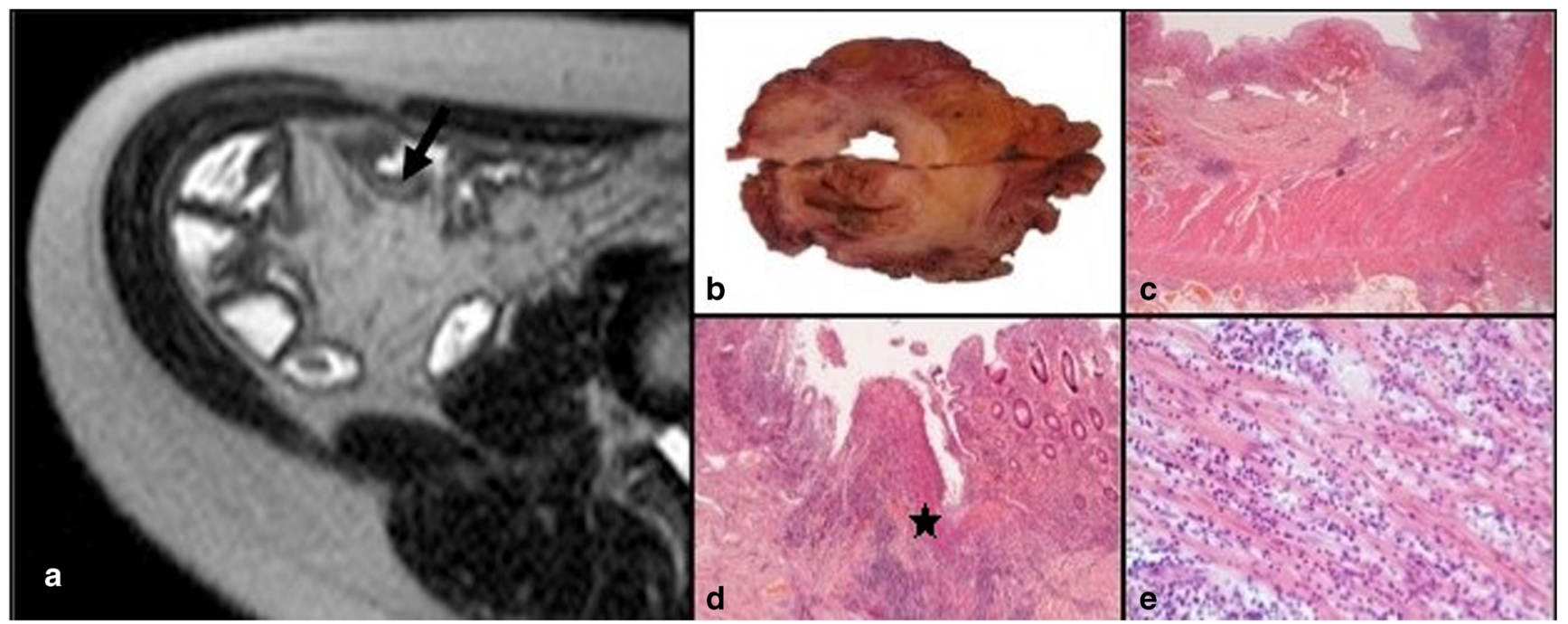

Fig. 3 Anastomotic ileal recurrent Crohn's disease with high skewness on MRTA and increased histological inflammation (AIS score of 7). a Axial T2-weighted coronal SSTSE through the neoterminal ileum (arrow) attracting a Crohn's disease activity score of 9 (large ROI) and skewness of 0.5346 (small ROI). b Coronal section of the bowel through the resection specimen matched to the site of sampling on MRI. c Full thickness H\&E stained histological section ( $\times 12.5$ magnification $)$ showing mucosal ulceration and transmural inflammation with lymphoid aggregates. d H\&E-stained histological section, $\times 100$ magnification) shows confluent mucosal ulceration. e H\&E-stained histological section $(\times 200$ magnification) taken from the region of the star in D shows an acute inflammatory cell infiltrate extending into the muscularis propria the small ROI matched with histology and so this association remains speculative.

The inverse correlation between total MRI CDA score and kurtosis is also interesting. Kurtosis is inversely related to the number of objects highlighted (whether bright or dark) and we can hypothesize that a greater number of highlighted objects reflects the more chaotic histology seen in active disease.

The total MRI score includes scores for contrast-enhanced sequences as well as extra mural signs such as perimural T2 signal which may influence how the total MRI activity score correlates with textural analysis in the T2-weighted sequence alone. Clearly, TA of contrast-enhanced T1-weighted images should be performed going forward. This was unfortunately not possible as the original MR enterography protocol utilised T1-weighted images acquired in the coronal plane only with non-isotropic voxels, impeding accurate TA. Another weakness was that we did not use fat-saturated T2-weighted images which were also not consistently available. It would be interesting to speculate if the data would be different if the fat signal from the bowel wall was nullified first, as this would perhaps have strengthened the association between TA parameters and histological inflammation.

Our study has other limitations. The sample size is small although reasonable for exploratory histopathological
Table 4 Summary of significant associations between texture features and mural MRI features for the large ROI

\begin{tabular}{lllll}
\hline Filter size (mm) & MRI feature & Textural parameter & Odds ratio (95\% CI) & $P$ value \\
\hline 2 & Mural thickness & MPP $^{\mathrm{a}}$ & $0.91(0.83,1.00)$ & 0.04 \\
2 & Mural T2 signal & Mean $^{\mathrm{a}}$ & $1.22(1.02,1.47)$ & 0.03 \\
& & Entropy $^{\mathrm{b}}$ & $3.16(1.44,6.95)$ & 0.004 \\
2 & Mural enhancement & MPP $^{\mathrm{a}}$ & $0.91(0.86,0.97)$ & 0.003 \\
3 & Mural T2 signal & Mean $^{\mathrm{a}}$ & $1.26(1.01,1.58)$ & 0.04 \\
& & Entropy $^{\mathrm{b}}$ & $2.76(1.20,6.37)$ & 0.02 \\
3 & Mural enhancement & MPP $^{\mathrm{a}}$ & $0.86(0.75,0.99)$ & 0.04 \\
& & Kurtosis $^{\mathrm{b}}$ & $0.59(0.41,0.84)$ & 0.004 \\
4 & Mural T2 signal & Mean $^{\mathrm{a}}$ & $1.28(1.02,1.61)$ & 0.03 \\
& & Entropy $^{\mathrm{b}}$ & $2.32(1.12,4.83)$ & 0.02 \\
5 & Mural enhancement & SD $^{\mathrm{a}}$ & $0.42(0.22,0.78)$ & 0.007 \\
& & MPP $^{\mathrm{a}}$ & $0.18(0.07,0.48)$ & 0.001 \\
\hline
\end{tabular}

${ }^{\text {a }}$ Odds ratio reported for a 50-unit increase in predictor variable

${ }^{\mathrm{b}}$ Odds ratio reported for a 1 -unit increase in predictor variable 


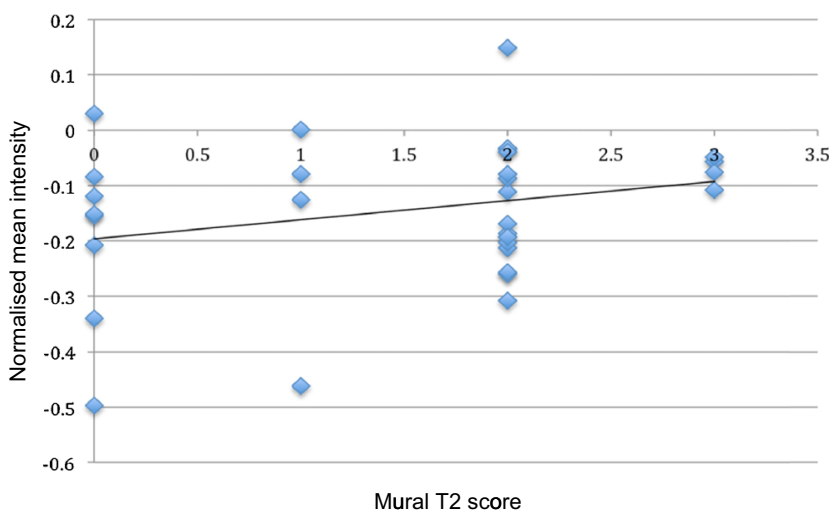

Fig. 4 Scatter plot and line of regression between normalised mean intensity and T2 MRI score (subjectively graded from 0 to 3 depending on intensity of mural T2 signal) at SSF 2 for the large ROI

imaging correlative research of a relatively novel technology. Using a patient cohort undergoing surgical resection risks spectrum bias since such patients will have more advanced disease than those newly diagnosed. However this is unavoidable if full-thickness histopathological correlates are required, but the results are only applicable to this patient cohort. Bowel wall is a relatively small structure compared to larger tumours where TA has been applied successfully and repeatedly. We were careful to restrict ROIs to the bowel wall, but bowel lumen or mesentery may have been included inadvertently. Of note, TA has been applied to very small structures such as knee cartilage and, by definition, the bowel is usually thickened significantly in Crohn's disease. The filtration-histogram TA approach is a key step towards extracting and quantifying texture features selectively at different filter sizes (SSF), related to tissue biology. Filtration at a scale above $2 \mathrm{~mm}$ minimizes the impact of image noise (more impacted at scales less than $2 \mathrm{~mm}$ ) and further normalization of relevant texture quantifiers (minimizing the impact of variation in MR acquisition parameters) makes our approach more robust. Nevertheless, the restricted size of bowel ROI limits the volume of texture data that can be derived, and for this reason we limited the range of filters used.

Whilst the MRI CDA is validated as a score of activity, evaluation of T2-weighted images is an important part of the score and so MRTA metrics based on T2-weighted images may perhaps be expected to correlate with the CDA. Whilst we attempted to normalize T2 signal to CSF, it would likely have been better to normalise the whole image as the relationship between texture values and initial image brightness, strictly speaking, is nonlinear. However other workers have successfully used MRTA in non-normalised T2-weighted images in breast cancer [28], so we feel our approach was reasonable.

A multivariate statistical analysis would be more meaningful in future studies with a larger sample size.
It is interesting to speculate how MRTA could be used in clinical practice. The underlying premise is that macroscopic imaging features are a marker for microscopic histological phenotype. Most work has been done in cancer, where TA parameters are associated with genetic mutation status, hypoxia and angiogenesis, and even long-term prognosis. The utility of TA on patient management, however, remains somewhat controversial with unresolved questions regarding reproducibly and robustness of the methods (reflected in part by the variability in the data of the current study). Perhaps the most likely role of MRTA in Crohn's disease would be as an objective marker of treatment response, where a measurable change in skewness for example acts as a biomarker for reduced histological inflammation. A logical next step would be to investigate MRTA before and after treatment.

Clearly with such a small sample, our conclusions are speculative at this stage, and a larger sample size may have produced a wide range of TA parameter correlation to histopathological activity grading.

The use of multiple regression analysis may produce spurious or chance associations. Just 15/120 large ROI correlations were indeed significant. Thus, our data must be viewed critically but merits future investigation.

\section{Conclusion}

Our preliminary data suggests that some MRTA parameters may be associated with histological and MRI activity scores. Skewness measured using MRTA at a 2-mm filter level is potentially associated with a histological CD activity score. Additional MRTA features including kurtosis and entropy may also be associated with a validated MRI activity score (Crohn's disease activity score). Such associations now need to be confirmed with larger sample sizes and appropriate statistical modelling to assess whether MRTA can act as an imaging biomarker of Crohn's disease actively.

Acknowledgments The scientific guarantor of this publication is Prof. Stuart A. Taylor. The authors of this manuscript declare relationships with the following companies: S.A.T. is a research consultant for Robarts plc, B.G. is part-employed within the Feedback plc; B.G. and K.M. are shareholders in the Feedback plc, a company developing and commercializing the TexRAD (www.texrad.com, part of Feedback Plc, Cambridge, UK) imaging texture analysis research software algorithm considered in this study.

This study has received funding by the National Institute for Health Research (NIHR) University College London Hospitals Biomedical Research Centre. S.A.T and S.H. are NIHR senior investigators. J.M.'s salary was supported by EU FP7 grant 270379 (vigor++).

TexRAD (www.texrad.com, part of Feedback Plc, Cambridge, UK) provided software for this study. The human tissue used for research was obtained from the HTA licensed UCL/UCLH biobank for health and disease (HTA Licence number 12055) approved by the National Research Ethics Committee, Leeds (east) Research Ethics Committee (Reference number 10/H1306/42). The biobank is funded by the 
National Institute for Health Research, UCLH Biomedical Research Centre, the UCL experimental Cancer Research Centre and the Cancer Research UK-UCL Centre. Mr. Paul Bassett, an independent medical statistician, kindly provided statistical advice for this manuscript. Institutional review board approval was obtained. The local research ethics committee (UCLH committee alpha) gave ethical approval and written informed consent was originally obtained from all participants. The institutional review board issued a waiver for re-consenting patients for additional data analysis. Written informed consent was waived by the institutional review board. Some study subjects or cohorts have been previously reported in Punwani S, Rodriguez-Justo M, Bainbridge A et al (2009) Mural inflammation in Crohn disease: location-matched histologic validation of MR imaging features. Radiology 252:712-720. doi:10.1148/2523082167. Methodology: retrospective.

Open Access This article is distributed under the terms of the Creative Commons Attribution 4.0 International License (http:// creativecommons.org/licenses/by/4.0/), which permits unrestricted use, distribution, and reproduction in any medium, provided you give appropriate credit to the original author(s) and the source, provide a link to the Creative Commons license, and indicate if changes were made.

\section{References}

1. Rimola J, Rodriguez S, Garcia-Bosch O et al (2009) Magnetic resonance for assessment of disease activity and severity in ileocolonic Crohn's disease. Gut 58:1113-1120

2. Rimola J, Ordás I, Rodriguez S et al (2011) Magnetic resonance imaging for evaluation of Crohn's disease: validation of parameters of severity and quantitative index of activity. Inflamm Bowel Dis 17:1759-1768

3. Steward MJ, Punwani S, Proctor I et al (2012) Non-perforating small bowel Crohn's disease assessed by MRI enterography: derivation and histopathological validation of an MR-based activity index. Eur J Radiol 81:2080-2088

4. Zappa M, Stefanescu C, Cazals-Hatem D et al (2011) Which magnetic resonance imaging findings accurately evaluate inflammation in small bowel Crohn's disease? A retrospective comparison with surgical pathologic analysis. Inflamm Bowel Dis 17:984-993

5. Makanyanga JC, Pendsé D, Dikaios N et al (2014) Evaluation of Crohn's disease activity: initial validation of a magnetic resonance enterography global score (MEGS) against faecal calprotectin. Eur Radiol 24:277-287

6. Tielbeek JAW, Lowenberg M, Bipat S et al (2013) Serial magnetic resonance imaging for monitoring medical therapy effects in Crohn's disease. Inflamm Bowel Dis 19:1943-1950

7. Miles KA, Ganeshan B, Hayball MP (2013) CT texture analysis using the filtration-histogram method: what do the measurements mean? Cancer Imaging 13:400-406

8. Ganeshan B, Miles KA (2013) Quantifying tumour heterogeneity with CT. Cancer Imaging 13:140-149

9. Ganeshan B, Panayiotou E, Burnand K et al (2012) Tumour heterogeneity in non-small cell lung carcinoma assessed by CT texture analysis: a potential marker of survival. Eur Radiol 22:796-802

10. Ganeshan B, Strukowska O, Skogen K et al (2011) Heterogeneity of focal breast lesions and surrounding tissue assessed by mammographic texture analysis: preliminary evidence of an association with tumor invasion and estrogen receptor status. Front Oncol 1:33
11. Miles KA, Ganeshan B, Griffiths MR et al (2009) Colorectal cancer: texture analysis of portal phase hepatic CT images as a potential marker of survival. Radiology 250:444-452

12. Miles KA, Ganeshan B, Rodriguez-Justo M et al (2014) Multifunctional imaging signature for V-KI-RAS2 Kirsten rat sarcoma viral oncogene homolog (KRAS) mutations in colorectal cancer. J Nucl Med 55:386-391

13. Weiss GJ, Ganeshan B, Miles KA et al (2014) Noninvasive image texture analysis differentiates K-ras mutation from pan-wildtype NSCLC and is prognostic. PLoS One 9, e100244

14. De Cecco CN, Ganeshan B, Ciolina M et al (2015) Texture analysis as imaging biomarker of tumoral response to neoadjuvant chemoradiotherapy in rectal cancer patients studied with 3-T magnetic resonance. Investig Radiol 50:239-245

15. Ganeshan B, Skogen K, Pressney I et al (2012) Tumour heterogeneity in oesophageal cancer assessed by $\mathrm{CT}$ texture analysis: preliminary evidence of an association with tumour metabolism, stage, and survival. Clin Radiol 67:157-164

16. Maccioni F, Staltari I, Pino AR, Tiberti A (2012) Value of T2weighted magnetic resonance imaging in the assessment of wall inflammation and fibrosis in Crohn's disease. Abdom Imaging 37: 944-957

17. Punwani S, Rodriguez-Justo M, Bainbridge A et al (2009) Mural inflammation in Crohn disease: location-matched histologic validation of MR imaging features. Radiology 252:712-720

18. Tielbeek JA, Ziech ML, Li Z et al (2014) Evaluation of conventional, dynamic contrast enhanced and diffusion weighted MRI for quantitative Crohn's disease assessment with histopathology of surgical specimens. Eur Radiol 24:619-629

19. Steward MJ, Punwani S, Proctor I et al (2011) Non-perforating small bowel Crohn's disease assessed by MRI enterography: derivation and histopathological validation of an MR-based activity index. Eur J Radiol 81:2020-2028

20. Borley NR, Mortensen NJ, Jewell DP, Warren BF (2000) The relationship between inflammatory and serosal connective tissue changes in ileal Crohn's disease: evidence for a possible causative link. J Pathol 190:196-202

21. Skogen K, Ganeshan B, Good C et al (2013) Measurements of heterogeneity in gliomas on computed tomography relationship to tumour grade. J Neurooncol 111:213-219

22. Danese S, Sans M, de la Motte C et al (2006) Angiogenesis as a novel component of inflammatory bowel disease pathogenesis. Gastroenterology 130:2060-2073

23. Chen G, Strzelecki M, Pang Q et al (2010) Textures in magnetic resonance images of the ischemic rat brain treated with an antiinflammatory agent. Clin Imaging 34:7-13

24. Nketiah G, Savio S, Dastidar P et al (2014) Detection of exercise load-associated differences in hip muscles by texture analysis. Scand J Med Sci Sports. doi:10.1111/sms.12247

25. Boutsikou K, Kostopoulos S, Glotsos D et al (2013) Texture analysis of articular cartilage traumatic changes in the knee calculated from morphological 3.0T MR imaging. Eur J Radiol 82:1266-1272

26. Mahapatra D, Schueffler P, Tielbeek J, Buhmann J, Vos F (2013) A supervised learning approach for Crohns disease detection using higher order image statistics and a novel shape asymmetry measure. J Digit Imaging 26:920-931

27. Tielbeek JA, Makanyanga JC, Bipat S et al (2013) Grading Crohn disease activity with MRI: interobserver variability of MRI features, MRI scoring of severity, and correlation with Crohn disease endoscopic index of severity. AJR Am J Roentgenol 201:1220-1228

28. Parikh J, Selmi M, Charles-Edwards G et al (2014) Changes in primary breast cancer heterogeneity may augment midtreatment MR imaging assessment of response to neoadjuvant chemotherapy. Radiology 272:100-112 